\title{
Balkanologie
}

Balkanologie Revue d'études pluridisciplinaires

Vol. VII, n 2 | 2003

Volume VII Numéro 2

\section{La problématique de l'Europe (re)découverte par le parti socialiste bulgare}

\section{Marta Touykova}

\section{(2) OpenEdition}

1 Journals

Édition électronique

URL : http://journals.openedition.org/balkanologie/505

DOI : $10.4000 /$ balkanologie. 505

ISSN : 1965-0582

Éditeur

Association française d'études sur les Balkans (Afebalk)

Édition imprimée

Date de publication : 1 décembre 2003

Pagination : 199-225

ISSN : 1279-7952

\section{Référence électronique}

Marta Touykova, «La problématique de l'Europe (re)découverte par le parti socialiste bulgare », Balkanologie [En ligne], Vol. VII, n² 2 | 2003, mis en ligne le 19 février 2009, consulté le 17 décembre 2020. URL : http://journals.openedition.org/balkanologie/505 ; DOI : https://doi.org/10.4000/ balkanologie. 505

(c) Tous droits réservés 


\title{
LA PROBLÉMATIQUE DE L'EUROPE (RE)DÉCOUVERTE PAR LE PARTI SOCIALISTE BULGARE
}

\author{
Marta Touykova*
}

" Il existe un consensus politique en Bulgarie sur la stratégie du pays en ce qui concerne l'orientation de la politique étrangère. L'adhésion à part entière à l'Union européenne et à l'OTAN bénéficie du soutien de toutes les forces politiques représentées à l'Assemblée nationale et de la vaste majorité de l'opinion publique bulgare. ${ }^{1}$ Cette affirmation de Georgi Părvanov, ancien leader du Parti socialiste bulgare (PSB) et actuel Président de la République de Bulgarie, n'était pas envisageable il y a seulement deux ans en Bulgarie. En effet, si un consensus politique en faveur de l'intégration européenne a émergé dès le début des années 1990, il faudra attendre l'an 2000 pour que le PSB se rallie au principe de l'intégration euro-atlantique. Par ailleurs, la thématique euroatlantique a été très présente dans le débat politique bulgare courant 2002 : la Bulgarie a obtenu une date pour son adhésion à l'UE (2007) et une invitation à rejoindre l'OTAN en 2004.

En fait, si l'idée que la Bulgarie a sa place dans une Europe unifiée et qu'elle doit se développer à son image a été admise et véhiculée par les élites politiques et a trouvé un écho dans la population ${ }^{2}$, les implications concrètes de ce processus de rapprochement ne faisaient pas l'objet d'un accord au sein de la classe politique. L'Europe désigne bien plus qu'un ensemble géographique, elle recouvre une multitude de dimensions et de significations - culturelles, politiques, institutionnelles, juridiques, économiques, sociales, etc. - attachées à l'Europe de l'Ouest, l'Occident, et qui recouvrent également les États-Unis intégrés via la dimension atlantique. Toutefois, de façon générale,

•IEP, Paris. (marta@club-internet.fr)

${ }^{1}$ Părvanov (Georgi), " Vers la stabilité en Europe du Sud-Est ", Socialist Affairs, 49 (4), 2002.

${ }^{2}$ L'intégration dans l'UE a toujours bénéficié d'un support plus important que l'adhésion à l'OTAN. 
la transition a été considérée comme un retour à l'Europe et les emprunts comme un passage obligé afin d'atteindre ce modèle. Le changement se présente comme un processus d'européanisation qui prend principalement deux directions : une convergence de la formalisation de la vie politique, économique et sociale du pays à travers l'adoption de règles, de normes, de modèles européens et un rapprochement en vue de l'intégration du pays dans l'Union européenne (UE) et l'OTAN. Ainsi, la (re)démocratisation a tendance à être présentée par les observateurs et les acteurs comme un retour vers l'Europe - entendue ici comme Occident et désignée par le couple euro-atlantique. Ce processus impulsé et entretenu par les élites politiques locales, avec le soutien de conseillers et d'experts étrangers, a été pris en charge par les partis politiques à la recherche de ressources légitimatrices. Les références à l'Europe et l'intégration européenne sont devenues des outils politiques à usage national et ont été intégrées dans la construction partisane et les relations inter et intra-partisanes. Or, la question de la construction partisane des organisations politiques qui ont émergé aux lendemains des changements de 1989 en Bulgarie se pose différemment selon qu'il s'agit d'une part, de partis nouvellement créés ou recréés (les partis historiques issus des parties interdits à partir de 1944) ou, d'autre part, des partis successeurs des anciens partis communistes. La légitimité de ces derniers, même s'ils ont participé au changement de régime et se sont ralliés aux règles du jeu démocratique, est fragile, ils doivent donner des gages de bonne conduite et prouver qu'ils ont rompu avec le passé. Cette exigence souligne toute l'ambiguité et la complexité du statut de parti successeur - il implique la simultanéité d'un processus de rénovation et d'un processus de conservation.

Parallèlement, l'émergence d'un paysage politique différencié rend visibles les tensions et les clivages sur lesquels se construisent les partis politiques. Dans les premières années des changements, la vie politique bulgare se caractérise par une grande instabilité et de fréquentes crises. Jusqu'en 2001, deux partis - le PSB, successeur de l'ancien parti communiste et l'Union des forces démocratiques (UFD), front anticommuniste issu de l'opposition - se sont succédé au pouvoir. L'année 2001 a été marquée par une double alternance inédite, le Mouvement politique Siméon II (MNSII), créé quelques mois seulement avant les élections par le dernier roi bulgare revenu d'exil, a remporté le scrutin législatif de juin, alors qu'en novembre, le leader du PSB, Georgi Părvanov, est devenu le premier Président socialiste de la Bulgarie post-communiste. Les années d'alternance au pouvoir entre le PSB et l'UFD se caractérisent par une idéologisation exacerbée sur l'axe communisme-anticommunisme qui est imposé comme grille de lecture de la vie politique bulgare. L'enjeu principal consiste à prouver la rupture avec l'ancien régime et à assurer l'irréversibilité du choix démocratique. L'européanisation est convoquée pour prouver cette rupture avec le passé, elle devient un enjeu sur lequel les 
partis doivent se positionner. La plupart des partis bulgares nouent des liens, établissent des échanges, reprennent des problématiques et des références idéologiques et se reconnaissent dans des homologues ouest-européens et tentent d'adhérer aux fédérations européennes de partis. Des distinctions entre partis pro-européens et partis anti- ou non-européens (qui mobilisent d'autres allégeances, identités et partenariats) s'établissent. En fait, c'est principalement la question de l'adhésion de la Bulgarie à l'OTAN qui a fait l'objet d'une confrontation vive entre les deux principaux partis bulgares. L'UFD a tenté de transformer la question de l'adhésion à l'OTAN, et plus précisément la réticence des socialistes, en ligne de fracture dans le paysage politique bulgare. Le refus du PSB était alors présenté comme le symptôme de l'incapacité du parti successeur à se réformer et à abandonner les allégeances du passé - il s'agissait de délégitimer ce parti.

Ce que cet article se propose d'étudier à travers la difficile construction partisane du PSB et de sa légitimation dans l'espace politique bulgare, ce sont les emprunts, l'instrumentalisation des liens avec l'Occident et notamment avec les partis sociaux-démocrates européens. Ce qui se joue, entre autres, à travers ces processus de mimétisme et de différenciation, c'est la (re)définition de la gauche bulgare. Une définition complexe à la fois en raison des difficultés internes et de l'hétérogénéité même du PSB, traversé par des courants divers et des tensions et qui reste marqué par un héritage communiste prégnant et stigmatisé par ses concurrents issus de la dissidence. Il s'agit de s'intéresser à l'adaptation et à la transformation de ce parti politique bulgare en référence au modèle européen. Ce processus comporte deux dimensions :

- l'adoption du modèle du parti de gauche européen -il s'agit du processus de normalisation du PSB, sa transformation en "parti comme les autres" qui prend la forme d'une social-démocratisation et se concrétise par des transferts idéologiques et programmatiques et une recherche de partenaires, amis et parrains européens et occidentaux;

- son ralliement à l'intégration euro-atlantique - sa transformation en parti capable et désireux de mener à bien l'intégration euro-atlantique de la Bulgarie. En effet, en tant que parti successeur, le PSB véhicule l'héritage d'une autre affiliation, son orientation pro-russe qui constitue un élément fondateur de son identité.

Avant d'analyser ces dimensions, il convient de revenir sur la thématique européenne dans la vie politique bulgare. 


\section{CONCEPTIONS / PERCEPTIONS DE L'EUROPE EN BULGARIE}

Le processus de passage à la démocratie, vécu et perçu par les acteurs comme une "voie" ou un "retour" vers l'Europe, réactualise dans le cas bulgare une vision de l'Europe comme symbole et modèle de modernisation ${ }^{3}$. La plupart des chercheurs bulgares s'accordent que le processus de changement politique et économique des années 1989 et d'intégration européenne constitue la troisième vague de modernisation-imitation pour la Bulgarie - la première étant celle du Réveil national et de la construction de l'État moderne bulgare et la deuxième l'industrialisation lancée dans les années 1930 et poursuivie par le régime communiste 4 . Au cours de ces phases de modernisation et de rattrapage, les élites bulgares ont érigé le modèle européen en objectif à atteindre. Il s'agit à la fois de (ré)intégrer un espace géographique commun après les périodes de séparation que sont l'intégration dans l'Empire ottoman ou dans le bloc soviétique, un espace politique qui partage les mêmes règles et normes et un espace économique. L'Europe est présentée comme une civilisation porteuse et synonyme de modernité, de changements (politiques, culturels, sociaux), de prospérité, de bien-être économique et de progrès (techniques). Il s'agit en fait d'une entité imaginaire, imaginée (d'ailleurs, les traditions nationales ne sont pas forcément différenciées) à laquelle est associé un ensemble de valeurs. On constate donc une réceptivité aux modèles étrangers, une aspiration des élites bulgares à l'européanisation du pays qui se concrétise par des stratégies de transferts et d'importation.

La relation par nature déséquilibrée entre la périphérie et le centre est également source de déceptions et de ressentiments. L'idéalisation d'un modèle européen crée des attentes importantes. Le désintérêt ou le rejet des parrains désirés peut provoquer la déception et le ressentiment. L'attraction souligne la différence et le retard bulgare et peut également susciter des inquiétudes et être présentée comme une menace potentielle pour l'identité et les intérêts du pays. D'ailleurs, le modèle européen n'est pas l'unique référence mobilisée par les élites bulgares. L'autre pôle d'attraction est constitué par la Russie, présentée par ses supporters comme le "double libérateur" (du "joug" ottoman et du fascisme). Sa place et son rôle dans la vie du pays à certains moments de l'histoire font l'objet d'un débat vif au sein de la classe politique divisée entre rus-

${ }^{3} \mathrm{Cf}$. notamment Dimitrov (Georgi), Bälgarija v orbitite na modernizacijata (La Bulgarie dans l'orbite de la modernisation), Sofia : Universitetsko izdatelstvo Sv. Kliment Oxridski, 1995 ; Daskalov (Rumen), " Obrazi na Evropa sred bălgarite " (Images de l'Europe parmi les Bulgares), in Meždu iztoka i zapada. Bälgarski kulturni dilemi (Entre l'orient et l'occident. Dilemmes culturels bulgares), Sofia : Lik, 1998 ; Nikova (Ekaterina), " La modernisation à travers l'intégration : la Bulgarie et l'Union européenne ", Transitions, 42 (1), 2001.

4 Dičev (Ivajlo), “ Evropa kato legitimicija "(L'Europe comme légitimation), Sociologičeski problemi, (12), 2000 , p. 7 . 
sophiles et russophobes. Le rapport à ces influences extérieures fait depuis le $\mathrm{XIX}^{\mathrm{e}}$ siècle l'objet de débats au sein des élites bulgares et de luttes entre pro- et anti-, -philes et -phobes, à propos du modèle le mieux adapté aux réalités locales et des parrains les mieux disposés envers le pays et le mieux à même de défendre ses intérêts.

\section{Les images de l'Europe dans une perspective historique}

L'image de l'Europe qui émerge en Bulgarie au cours du XIX ${ }^{e}$ siècle est construite, diffusée et renouvelée par des passeurs - intellectuels, hommes politiques, voyageurs, étudiants qui ont suivi une formation en Europe. Les contacts avec l'Europe se développent et la connaissance de ses mœurs, habitudes, de son histoire commence à être diffusée. Ces contacts sont par ailleurs des ressources socialement valorisées, sources de prestige et d'influence. L'attraction exercée par l'Europe se concrétise par la transposition des règles du jeu politique. Les bâtisseurs du jeune État bulgare sont à la recherche de modèles et d'affiliations et ils se tournent vers l'Europe - l'adoption de la monarchie constitutionnelle et la lettre de la Constitution bulgare de 1879 témoignent de ces emprunts aux modèles européens. Ces transferts sont simultanément encouragés par les "Grandes Puissances" qui parrainent la construction de l'État bulgare.

Le régime socialiste va tenter de créer deux blocs antagonistes et séparés. Il va essayer d'instaurer une nouvelle séparation entre l'Europe (de l'Ouest) et la Bulgarie et de déplacer le pôle d'attraction de l'Europe vers l'URSS en exploitant le fond de russophilie présent chez certaines élites et couches de la population. Non seulement, ce discours ne va pas véritablement prendre, ni réussir à construire une frontière imperméable entre les deux blocs, mais il va contribuer à développer en réaction une image idyllique de l'Ouest comme terre de libertés et d'abondance. Image d'autant plus magnifiée que les contacts avec l'Europe et l'information en provenance de l'Ouest sont réduits même si les relations diplomatiques sont maintenues ou rétablies avec certains pays occidentaux.

Le passage vers la démocratie et l'économie de marché marquent le rejet de ce modèle alternatif incarné par l'Union soviétique et le ralliement au modèle et aux valeurs européens. Les “autres" Européens s'identifient et se reconnaissent dans l'Europe puis ils tentent de l'imiter, de la transposer - de réaliser une réunification symbolique du continent. Ce mouvement s'accompagne d'un travail de redécouverte du passé européen des anciens pays de l'Est. D'ailleurs, l'expression pays de l'Est qui marquait la division idéologique est remplacé par une dénomination qui se veut plus neutre : Europe centrale et orientale. En Bulgarie, comme dans les autres PECO, on assiste à nouveau à l'émergence d'un discours sur l'Europe. Ce discours comporte plusieurs facettes et révèle les différentes dimensions projetées dans la notion Europe. Il met en 
évidence des tensions entre une appartenance ressentie et déclarée à l'Europe et un processus d'intégration soumis à des conditions et à des contraintes concrètes qui peuvent devenir des obstacles sur le chemin du retour.

Ainsi, pour reprendre la classification de F. de la Serre, C. Lequesne et J. Rupnik 5 , on peut établir que l'Europe est présentée comme un choix de civilisation, un choix politique, économique et de sécurité.

L'Europe est une civilisation, une culture, à laquelle la Bulgarie appartient de droit en dépit des arrachements consécutifs à son intégration imposée dans l'Empire ottoman puis l'Union soviétique. La Bulgarie a des mérites dans l'édification de la civilisation européenne (l'alphabet cyrillique). La Bulgarie est chrétienne. "Ce véritable consensus [sur la priorité que la Bulgarie accorde à son intégration dans les structures économiques et politiques européennes] reflète le profond sentiment national d'appartenance à la même communauté de valeurs et d'aspirations humaines, au même patrimoine historique et culturel. " ${ }^{6}$ L'appartenance à l'Europe est un "choix de civilisation" selon les termes du Président Stojanov.

Le retour à l'Europe exprime également un choix politique revendiqué par tous les partis bulgares, celui de la démocratie. Jeliou Jelev souligne bien cet enjeu : "l'intégration aux organisations européennes et euro-atlantiques est porteuse d'une dynamique démocratique. Notre adhésion à celles-ci rendra irréversibles les processus démocratiques à l'intérieur du pays "7. En effet, l'intégration dans le concert européen impose des contraintes en matière de respect des règles démocratiques ce qui permet en quelque sorte d'arrimer solidement la démocratie.

Le choix du modèle européen comporte également une dimension économique - l'adoption de l'économie de marché et l'espoir qu'elle apportera la prospérité au pays - et une dimension en matière de sécurité extérieure avec la nécessité de réorienter la politique étrangère bulgare. C'est sur ces deux questions - le modèle économique et l'adhésion de la Bulgarie à l'OTAN - que les positions des deux principaux partis politiques bulgares ont différé.

Ce sentiment d'appartenance à une communauté de culture et de partage d'un ensemble de valeurs favorise la capacité à se reconnaître dans les modèles politiques européens, il nourrit le consensus européen. Dans cette optique, les emprunts ne sont pas considérés a priori comme la transplantation de

\footnotetext{
5 Serre (Françoise de la), Lequesne (Christian), Rupnik (Jacques), L'Union Européenne ouverture à l'Est ?, Paris : PUF, 1994 .

${ }^{6}$ Gotev (Guéorgui), "Bulgarie - Union Européenne : des efforts concertés pour préparer l'adhésion ", Les enjeux de l'Europe, (15), 1994 (l'auteur était alors Premier secrétaire de la mission bulgare auprès de l'UE).

7 Jelev (Jeliou), La Bulgarie terre d'Europe. La politique étrangère de la Bulgarie postcommuniste, Paris : éditions Frison-Roche, 1998, p. 69.
} 
quelque chose d'extérieur ou d'étranger, mais comme un rattrapage. Cette perception entretient l'illusion qu'il suffit d'exprimer son souhait de revenir dans la famille européenne pour y être admis et que cette admission doit s'accompagner de gestes symboliques, d'un traitement préférentiel (un plan Marshall pour l'Est). Or l'intégration européenne est soumise à des règles et à des procédures précises qui révèlent l'aspect contraignant et conditionnel de ce retour. En effet, l'intégration européenne et euro-atlantique implique un changement démocratique interne (juridique et institutionnel) comme condition nécessaire $^{8}$. Ce processus impose des règles et des transferts (l'acquis communautaire) concrets et contraignants. La contrainte s'exerce au travers de sommets, de décisions, de rapports périodiques, de feuilles de route mais également de coopérations, d'aides, d'accès aux fonds structurels de pré-adhésion.

De facto, le processus d'intégration met en évidence les décalages, les différences entre l'Europe et la Bulgarie et contribue à creuser les frontières (avant de pouvoir les effacer). Ce qui réactive le sentiment de division de l'Europe et d'exclusion. Ivailo Znepolski l'exprime par une image forte, pour lui le rideau de fer a été remplacé par un " rideau de normes et de régulations européennes " 9 . Maria Todorova dénonce l'émergence d'une " rhétorique de l'exclusion " qui s'appuie sur une image négative des Balkans et réactive des stéréotypes et des divisions de l'Europe $^{10}$. D'ailleurs, comme le souligne l'historienne Iskra Baeva,

la réflexion, exprimée à travers l'appel communément admis pour une " voie vers l'Europe " montre que les Européens de l'Est se définissent comme extérieurs à l'Europe, tout en ressentant douloureusement la façon dont ils sont définis par les autres : Orient ou frontière de la civilisation européenne. (...) Cette ambition perpétuelle de se comparer pour prouver son appartenance à l'Europe, conduit facilement à adopter de façon non critique de modèles non conformes aux données et traditions locales. ${ }^{11}$

Ces réflexions incitent à s'interroger sur la transposition de modèles qui, même s'ils sont perçus comme proches et sont acceptés, n'en restent pas moins importés, ainsi que sur la dimension téléologique des changements. De fait, il ne suffit pas seulement de rejoindre l'Europe en pensée et dans les intentions pour effacer le passé mais de mettre en place une série de changements concrets.

\footnotetext{
${ }^{8}$ Cf. notamment Rupnik (Jacques), " Eastern Europe : the international context ", Journal of Democracy, 11 (2), 2000 ; Roger (Antoine), « L'incidence de la contrainte externe sur le positionnement des partis politiques en Europe centrale et orientale ", in De Waele (Jean-Michel), éd., Partis politiques et démocratie en Europe centrale et orientale, Bruxelles : éditions de l'Université de Bruxelles, 2002.
}

9 Reuters, 26/10/99 (cité par Central European Review, (19), 1999).

${ }^{10}$ Todorova (Maria), " La Bulgarie entre le discours culturel et la pratique politique n, Politique étrangère, 63 (1), printemps 1998.

${ }^{11}$ Baeva (Iskra), " Bălgarskijat prexod sled $1989 \mathrm{v}$ istoričeski kontekst " (La transition bulgare après 1989 dans un contexte historique), Istoričeski pregled, 52 (1), 1996, p. 91. 


\section{L'Europe saisie par les élites politiques}

Le retour vers l'Europe est un processus essentiellement impulsé par le haut, ce sont les élites politiques qui sont à l'origine du discours sur l'Europe et qui sont les agents de l'européanisation. Ce processus se traduit notamment par :

- l'emprunt des règles du jeu, des normes en vigueur dans les vieilles démocraties européennes au niveau des institutions politiques, économiques, de la construction des partis politiques. Les emprunts et les influences étrangères sont visibles notamment au moment de l'adoption de la nouvelle Constitution bulgare rédigée, comme celles d'autres PECO, avec l'aide d'experts étrangers afin de recueillir le meilleur des textes étrangers. Les transferts reposent sur la croyance en l'efficacité d'un mécanisme - la démocratie constitutionnelle, l'économie de marché, etc. - pour résoudre les problèmes auxquels doit faire face la Bulgarie ;

- une réorientation de la politique étrangère, notamment en direction des organisations multilatérales et de coopération (dont l'UE et l'OTAN en premier lieu). La question du nouveau système de sécurité européen se pose avec acuité et surtout celle de l'adhésion ou non à l'OTAN. Le désir d'adhérer aux structures européennes - CEE/UE, Conseil de l'Europe, mais également l'OTAN marque un rétrécissement du sens du retour à l'Europe désormais entendu comme adhésion à l'UE et à l'OTAN.

La majorité des partis bulgares se réclame de valeurs européennes et recourt à des références européennes dans leur processus de construction partisane. Le discours sur l'Europe participe au travail de légitimation et d'identification des acteurs politiques. Les partis politiques - nouveaux ou réformés - en quête de rupture avec le passé et de ressources légitimatrices, de filiations et de parrainages convoquent l'Europe et les Européens. La plupart des organisations politiques se réclament d'une identité européenne, se construisent ou se réforment à l'image de leurs homologues des vieilles démocraties, certains affichent même la référence européenne dans leurs labels : la plate-forme "Voie bulgare vers l'Europe " (créée au sein du PCB fin 1989), le Parti pour les ÉtatsUnis d'Europe et du monde, l'Euro-gauche. Ces emprunts contribuent à l'émergence d'un système de partis qui présente des similitudes visibles (à travers les labels, les positionnements sur un axe gauche-droite, etc.) avec le système de partis ouest-européen et ce en dépit de spécificités locales (clivages spécifiques aux PECO, partis ethniques). Cette convergence est facilitée par l'intervention de formateurs et d'experts européens qui accompagnent et orientent les changements politiques. Les contacts avec des hommes politiques occidentaux sont mis en valeur, leur soutien est recherché et affiché parce qu'il est censé attester de cette proximité par l'attribution de brevets d'apprentissage ou de trans- 
formation réussie. De même, les principaux partis politiques bulgares se déclarent en faveur de l'adhésion à l'UE et, depuis deux ans, également à l'OTAN. Le mouvement vers l'Europe n'est pas uniquement un désir spontané d'inclusion, il est également impulsé par la contrainte externe exercée par des interlocuteurs concrets, des organisations et des bailleurs de fonds qui conditionnent l'octroi d'aides, de coopération, d'adhésion au respect de certaines règles. De ce fait, toute force politique se trouve confrontée au choix européen et sollicite des soutiens extérieurs. Les exigences de l'Europe, les sacrifices qu'elle demande sont d'ailleurs parfois utilisés pour justifier des décisions politiques - ils fonctionnent alors comme des arguments d'autorité, " l'Europe le veut ". Toutefois, la défaite électorale de l'UFD a démontré qu'un parti peut perdre les élections malgré un bilan jugé positif par les principaux partenaires européens.

En Bulgarie, où le clivage communiste / anticommuniste est resté prégnant, le thème européen a été convoqué pour compléter et actualiser ce clivage, les deux grands partis qui se situent sur cet axe entrent en concurrence pour obtenir le statut de parti authentiquement européen ou pour stigmatiser leur adversaire en le qualifiant d'anti-européen. Les deux principaux partis bulgares - l'UFD et le PSB - se sont saisi de l'Europe pour définir leur identité allant parfois jusqu'à réactualiser les querelles entre -philes et -phobes. En fait, quand ils revendiquent leur caractère européen, qu'ils se présentent comme les sujets de l'européanisation, ces partis revendiquent non seulement le statut de démocrates et de progressistes, ils s'érigent également en modernisateurs. L'obtention du statut " européen ", du rôle moteur dans le processus d'intégration européenne, est source de rétributions symboliques mais également matérielles. L'UE se matérialise à travers des projets concrets, des programmes, des fonds (Phare, Ispa, Sapard) qui donnent lieu à des appropriations locales. Ainsi comme le montrent certaines recherches menées en Bulgarie, les maires socialistes font un apprentissage rapide et réussi des mécanismes de financements européens car ils trouvent dans ce dispositif un moyen de s'émanciper du centre politique dirigé par leurs concurrents de l'UFD (1997-2001). L'enjeu européen est intégré de façon croissante au jeu des acteurs locaux dont le ralliement européen peut précéder celui de leur propre parti ${ }^{12}$.

Cependant, l'Europe ne se prête pas facilement à la monopolisation parce qu'elle est multiple et plurielle - il y a multiplicité d'États, de courants politiques et donc de parrains potentiels et de modèles qui peuvent être adoptés. Finalement la " voie vers l'Europe " fait l'objet d'un consensus parmi les forces

\footnotetext{
${ }^{12} \mathrm{Cf}$. notamment Angelidou (Aliki), « Programmes européens, pratiques locales : les projets de développement régional face aux enjeux politiques n, communication présentée au colloque L'Europe à l'épreuve de l'élargissement : modèles européens et pratiques locales, Paris : MSH, 25-27 octobre 2000 ; Kabakčieva (Petja), " Postrojavane na "Evropa" ili priobštavane kăm Evropa " (Construction de l'Europe ou intégration à l'Europe), Sociologičeski problemi, (1-2), 2000.
} 
politiques bulgares, même si toutes ne se reconnaissent pas dans la même Europe. Toutefois, à partir du moment où le processus d'adhésion se met en place, cela crée une incitation pour les partis politiques à coopérer au nom de l'intérêt national. Le fait que le retour à l'Europe soit prôné par la majorité des partis politiques bulgares, favorise la convergence de leurs programmes - la démocratie politique, l'économie de marché, des relations internationales pacifiques et stables sont des thèmes présents dans les programmes de la majorité des partis bulgares. Toutefois le thème européen contribue également à différencier le paysage politique bulgare. Antony Todorov identifie quatre modes de différenciation : institutionnelle, politique, axiologique et gnoséologique $^{13}$. Effectivement, les tentatives d'appropriation et d'insertion du thème européen dans les luttes politiques bulgares et la concurrence que se livrent les acteurs politiques pour s'ériger en porte-parole et représentant de l'idée européenne contribuent à légitimer le pluralisme politique et à imposer les typologies et modes de classements européens (par exemple tous les débats sur la classification gauche - droite en Bulgarie).

En somme, l'Europe est synonyme de retour à la normalité - aux standards que sont la démocratie politique et l'économie de marché - et l'européanisation comporte plusieurs dimensions, culturelle, sociale, économique et politique. Il est impossible d'explorer toutes ces facettes, il s'agira donc ici d'étudier le rapport d'un des partis politiques bulgares avec l'Europe, tout d'abord sous l'angle de sa transformation interne, le PSB peut-il être considéré comme un parti européen, c'est-à-dire un parti "comme les autres" ?

\section{LE PSB - UN PARTI EUROPÉEN ?}

L'européanisation est perceptible au niveau du processus de transformation du parti successeur, elle se traduit par une convergence idéologique et programmatique avec les homologues européens, par des références explicites et des emprunts visant la normalisation et la standardisation du PSB. Il est en effet devenu membre de plein droit de l'IS en octobre 2003. Les élites du PSB essayent de conformer le processus de rénovation de leur parti à l'image qu'ils se font et qu'on leur donne d'un parti de gauche moderne. Il l'est également à travers la recherche de la reconnaissance par les homologues européens et de l'adhésion aux fédérations de partis.

13 Todorov (Antony), " The role of political parties in the Bulgaria's accession to the EU ", Center for the Study of Democracy Reports, (5), 1999, p. 10. 


\section{Un parti réformé - du point de vue idéologique, organisationnel et du point de vue de la pratique du pouvoir}

Dans les mois qui ont suivi les changements politiques, la plupart des partis communistes d'Europe de l'Est (à l'exception notable du Parti communiste de Bohême-Moravie) ont adopté un nouveau nom et entrepris, avec plus ou moins de facilité et de succès, une marche vers la social-démocratisation ${ }^{14}$. À partir de fin 1989 , le système politique bulgare connait une transformation rapide sous la pression de la rue et de l'UFD, le gouvernement communiste tente de limiter sa portée mais finit par accepter les réformes. L'abolition du rôle dirigeant du PCB est adoptée par le Parlement communiste en décembre 1989. Les principaux changements des règles du jeu politique sont décidés et négociés entre le PCB/PSB et l'UFD au cours des travaux de la Table ronde (janvier-mai 1990) avec notamment la reconnaissance du multipartisme et la décision de rédiger une nouvelle Constitution par une assemblée ad hoc - la Grande assemblée nationale - qui sera élue en juin 1990.

Sous l'impulsion des réformateurs qui ont procédé à l'éviction de Todor Zivkov du pouvoir, le PCB entame un processus de rénovation. La nouvelle direction du parti procède à des aggiornamentos : abandon du principe de la dictature du prolétariat, du centralisme démocratique, du système de parti unique, de la fusion entre le parti et l'État ; ralliement à l'économie de marché et reconnaissance des élections libres et concurrentielles comme mode unique d'accès au pouvoir. Dès lors, deux options sont envisageables : légitimer l'aile réformiste au sein d'une nouvelle structure politique ou bien tenter de sauvegarder le parti en l'état, en tant qu'organisation unie et stable capable de survivre et de peser dans le jeu politique. C'est la seconde solution, associée au premier leader du parti, Alexandăr Lilov ${ }^{15}$, qui sera adoptée. La décision de changer le nom du parti sans le dissoudre est approuvée par le vote des adhérents : $86,7 \%$ des 726 ooo votants se prononcent en faveur du nouveau nom " Parti socialiste bulgare ". Cette décision officialise cette transformation idéologique et la continuité de l'organisation. L'absence de rite de fondation spécifique, même motivée par la nécessité d'être rapidement opérationnel dans la perspective des élections fondatrices, a mis l'accent sur la continuité et sur l'adaptation plutôt que sur une rénovation plus radicale. Le PSB s'engage dans un processus de rénovation tout en étant le successeur de l'ancien parti unique. Comme le note Dobrin Kanev, " il existe une relation difficile et ambiguë entre

${ }^{14}$ Cf. notamment Dauderstädt (Michael), Gerrits (André), Markus (György G.), eds., Troubled transition. Social democracy in East-Central Europe, Bonn / Amsterdam : Friedrich Ebert stiftung / Wiardi Beckman Stichting / Alfred Mozer Stichting, 1999.

${ }^{15}$ Lilov est élu à la tête du PSB lors du $14^{e}$ Congrès en 1990, Videnov lui succédera au cours du $40^{e}$ Congrès en décembre 1991, il démissionnera en décembre 1996. Părvanov a ensuite présidé le Parti socialiste. Élu Président de la République en novembre 2001, il vient d'être remplacé par Sergei Stanišev. 
le processus de "succession" qui peut concerner la majeure partie de l'héritage, et le processus de "rénovation", qui implique, par définition, une prise de distance claire par rapport au passé communiste $»^{16}$. Une réforme organisationnelle est mise en place et la nouvelle direction procède à un renouvellement des élites au sein des instances exécutives du parti. La transformation se traduit également par l'adoption de nouvelles références programmatiques.

La volonté de s'inscrire dans une famille de la gauche européenne est perceptible à travers les orientations programmatiques du PSB. Le changement de l'idéologie du parti semble s'orienter vers la social-démocratie avec l'adoption du Manifeste pour le socialisme démocratique au cours du congrès de rénovation $\left(14^{\mathrm{e}}\right.$ Congrès du PCB, janvier-février 1990) - orientation confirmée au cours du premier congrès du parti renommé ( $39^{\mathrm{e}}$ Congrès du PSB, septembre $1990^{17}$ ) par la Plateforme pour la rénovation et la transformation du PSB en parti moderne du socialisme démocratique. C'est lors du congrès suivant $\left(40^{\mathrm{e}}\right.$ Congrès, décembre 1991) qu'est prise la décision de lancer un débat et de rédiger un programme d'action du parti. Le programme, intitulé " Nouveaux temps, nouvelle Bulgarie, nouveau PSB ", est adopté au cours du $41^{\mathrm{e}}$ Congrès en juin 1994 puis soumis au vote des adhérents. Il confirme " l'adoption de la trinité du socialisme démocratique... : liberté, justice sociale et solidarité $»^{18}$ et s'inspire de deux déclarations de l'Internationale socialiste : la déclaration adoptée à Francfort en 1951 sur les " Buts et tâches du socialisme démocratique " et la déclaration de principes de l'Internationale adoptée en 1989 à Stockholm. Le document présente le PSB comme une composante de la gauche européenne et mondiale ${ }^{19}$.

L'adoption d'une déclaration de principes ouvertement social-démocrate, peut fournir une fausse piste en suggérant que le processus de transformation du parti est achevé et l'identité du PSB gelée. L'inspiration et la lettre du texte, la succession de dates clés occultent les hésitations et les désaccords qui ont émaillé la mutation du parti. En fait, le ralliement à la social-démocratie est loin de faire l'unanimité au sein du parti, traversé par des courants hétérogènes qui couvrent l'ensemble de la palette de gauche. Au début des changements, le projet de social-démocratisation du PSB était porté par plusieurs mouvements (dont certains ont eu une existence éphémère), mais c'est l'Union pour la démocratie sociale créée en 1991 qui a été la plus active. L'UDS militait en vain pour que la question du rôle et du développement du parti soit réelle-

\footnotetext{
${ }^{16}$ Kanev (Dobrin), " La transformation du parti communiste bulgare ", in De Waele (Jean-Michel), èd., op. cit., p. 83

${ }^{17}$ Le PSB revient à la numérotation des congrès remontant à 1891 (cf. plus bas).

${ }^{18}$ Programme " Nouveaux temps, nouvelle Bulgarie, nouveau PSB ", p. 22

19 Ibid., p. 75
} 
ment tranchée au moment de l'adoption du nouveau programme afin qu'un engagement éventuel vers la social-démocratisation ne reste pas lettre morte. Les divergences et la volonté des directions successives de donner la priorité à la cohésion du parti ont conduit à l'éloignement progressif de la lettre du programme. Face à ce blocage, plusieurs personnalités réformatrices, dont des membres de l'UDS, ont quitté le parti. En février 1997, ils fondent ensemble le parti Eurogauche. Il s'agit de la seconde grande vague de départs de réformateurs. En fait, la résistance à la social-démocratisation a été portée par les deux premiers leaders du parti, Lilov et Videnov, qui ont tenté de privilégier une voie spécifique, ni communiste, ni sociale-démocrate. La voie de la "gauche moderne" dont la définition est restée floue, mais qui revenait à un refus de la transformation plus radicale du parti et de l'imitation sans condition des modèles sociaux-démocrates européens.

Le changement d'identité et de références vise d'abord à se démarquer du modèle communiste en forgeant une identité et une légitimité en résonance avec les nouveaux défis, c'est aussi une façon de rejeter le clivage mauvais (ex-) communistes / bons dissidents sur lequel le parti se constitue en proposant un nouveau projet qui permet de donner un sens à sa présence dans le paysage politique et de prouver son allégeance au nouveau système. La dimension stratégique est une composante importante dans ce revirement. La rénovation, stimulée à la fois par la contrainte interne et externe, devenait une condition de sa transformation en partenaire et en concurrent politique crédible et légitime et donc une condition de sa survie politique. L'adoption de normes et de valeurs différentes de celles véhiculées par son prédécesseur communiste relève d'une certaine stratégie d'imitation. Il s'agit de l'importation d'un modèle étranger, celui des partis socialistes et sociaux-démocrates ouest-européens, mais aussi de la redécouverte d'un héritage national refoulé (les sources de la gauche bulgare). Le Parti socialiste bulgare disposait d'une légitimité historique en se revendiquant d'une tradition social-démocrate bulgare puisqu'il est issu d'une des branches du Parti social-démocrate bulgare créé en 1891 par Dimităr Blagoev. Ce parti a connu une scission en 1903, deux mouvements s'étaient créés : le socialisme étroit et le socialisme large. Le Parti communiste bulgare, créé en 1919, était issu du mouvement du socialisme étroit. Les documents du PSB réactualisent cette filiation avec les pères et les principes fondateurs en proclamant le PSB héritier du parti créé en 1891 et en adoptant une numérotation des congrès qui remonte à cette date. Même si cette revendication reste cohérente, adhérer à un modèle stigmatisé sous le régime communiste ne va pas de soi pour un parti successeur. En effet, la social-démocratie était pour beaucoup d'anciens communistes un terme péjoratif et infamant, susceptible de provoquer des résistances chez les adhérents de longue date ${ }^{20}$. De surcroît, 
le PSB doit partager cette filiation avec le Parti social-démocrate "historique" (PSDB) reformé en décembre 1989. Ce parti, même s'il a échoué à devenir un acteur puissant dans la vie politique (comme d'autres partis "historiques" des PECO d'ailleurs ${ }^{21}$ ), peut aisément, du fait de sa continuité idéologique et de son appartenance à l'Internationale Socialiste (IS), se prévaloir d'incarner la socialdémocratie authentique. Ainsi, le label social-démocrate était pris. Cette concurrence a freiné l'objectif du PSB de s'ériger en porte-parole et représentant crédible de ces valeurs dans le paysage politique bulgare.

La référence à la social-démocratie est surtout une façon d'énoncer le changement. En fait, les débats relatifs à la social-démocratisation ont catalysé des visions différentes de la profondeur de la réforme du parti et donc de la rupture avec le passé. Définir la social-démocratisation comme un objectif revient à prôner un changement radical et à opter pour une identification explicite. C'est l'échec patent du gouvernement socialiste de Videnov (janvier 1995décembre 1996) qui ne réussit pas à doter le PSB d'une crédibilité de l'exercice du pouvoir, et la grave crise politique et économique qui aboutit au retrait du PSB du pouvoir et à sa lourde défaite électorale en juin 1997, qui contribue à relancer le processus de transformation du PSB. La lourde défaite électorale, l'isolement interne et international du parti et l'émergence d'une concurrence crédible à gauche ainsi que l'arrivée à la tête du parti de nouveaux leaders ont joué un rôle fortement incitatif pour le changement du PSB. La nouvelle équipe dirigeante du PSB a abordé certaines questions taboues jusqu'alors - clarification de l'identité du parti, recherches d'alliés (y compris chez les anciens transfuges du PSB), ralliement à l'OTAN. La réactivation des contacts avec l'IS et le PSE joue une place centrale dans cette relance de la construction-transformation partisane du PSB.

\section{Un parti reconnu par ses analogues / homologues européens. L'attente d'un brevet de légitimité}

L'adoption de références idéologiques avalisées par l'Europe, surtout quand elle n'est pas corroborée par une pratique gouvernementale adéquate, ne suffit pas à attester de l'appartenance à la famille politique européenne. Les documents programmatiques d'un parti peuvent rester lettre morte alors que l'adhésion à une organisation semble une preuve bien plus visible du ralliement à des valeurs communes et de l'appartenance à une famille politique européenne. Si la recherche du parrainage des partis des vieilles démocraties est commune à la plupart des partis politiques bulgares, dans le cas du parti successeur, elle revêt une importance beaucoup plus grande. Ce parrainage équi-

${ }^{21} \mathrm{Cf}$. Waele (Jean-Michel de), " Le retard social-démocrate en Europe centrale et balkanique " n. in Delwit (Pascal), Waele (Jean-Michel de), éds., La Gauche face aux mutations en Europe, Bruxelles : Éditions de l'Université de Bruxelles, 1993. 
vaut à l'octroi d'un brevet de changement (de non-communisme) et de légitimité, il semble fonctionner comme un argument d'autorité : " ces affiliations sont le sceau de l'approbation finale en termes de reconnaissance internationale comme de social-démocratisation $n^{22}$. Pour le PSB, le stade suprême de cette reconnaissance comme un "ami politique" 23 par les homologues européens devient l'adhésion souhaitée à l'Internationale socialiste et au Parti des socialistes européens. Elle permettrait d'attester la proximité idéologique et programmatique du PSB avec ses homologues des vieilles démocraties et serait également source de dividendes dans la compétition politique. La coopération transnationale des partis contribue à diffuser dans la vie politique bulgare les modèles européens et favorise la convergence, elle a également un impact sur la structuration interne des partis et sur la compétition inter-partisane. La direction du PSB est consciente de l'impact intérieur et des effets politiques d'une éventuelle adhésion à l'IS et médiatise les relations avec les fédérations de partis.

En orientant les changements programmatiques dans une certaine direction et/ou en stimulant les clarifications conceptuelles au sein des partis candidats, les parrains peuvent influencer les équilibres internes au sein des partis. Dans le cas du PSB, la reconnaissance internationale serait le couronnement de la stratégie de social-démocratisation du PSB portée par Părvanov et Stanišev. Les liens avec des "amis politiques" contribuent, par un processus de contamination, à la consolidation d'une identité sociale-démocrate du PSB. L'officialisation de son appartenance à la famille de la gauche sociale-démocrate consoliderait l'identité du parti et mettrait fin aux querelles sur l'identité du parti en donnant une prime à l'aile sociale-démocrate.

Les recherches de parrainage attisent la concurrence entre partis politiques nationaux de même obédience pour l'obtention du label européen mais peuvent également favoriser la coopération entre eux. C'est une orientation qui a été explicitement promue par le PSE et l'IS en Bulgarie, c'est sous leur impulsion que les différentes composantes de la gauche bulgare ont commencé à coopérer. Le soutien extérieur est fréquemment mobilisé dans les luttes interpartisanes. Jusqu'aux dernières élections, seule l'UFD pouvait se prévaloir avec succès du soutien occidental. Issue de la dissidence et associé au changement démocratique, l'UFD, membre du Parti populaire européen (PPE) depuis $1998^{24}$,

\footnotetext{
${ }^{22}$ Kanev (Dobrin), art. cit., p. 94

${ }^{23}$ Devin (Guillaume), "L'internationale socialiste en Europe centre-orientale : définition et rôle des "amis politiques" n, in Delwit (Pascal), Waele (Jean-Michel de), op. cit.

24 En novembre 1997, le Congrès de Toulouse du PPE décide de rendre possible l'adhésion de partis des pays candidats. L'adhésion de 4 partis, dont l'UFD, est approuvée lors d'une réunion du Bureau politique du PPE en mars 1998. Nadežda Mihailova, actuelle présidente de l'UFD, est vice-présidente du PPE depuis 1999.
} 
avait réussi à monopoliser le soutien des principaux acteurs européens. Pour le PSB la mobilisation de cet argument est relativement récente - elle a connu un développement lors des campagnes législatives et présidentielles de 2001. À son retour du congrès du PSE (Berlin, 7-8 mai 2001), Georgi Părvanov a annoncé avoir obtenu le "soutien catégorique" du parti ajoutant que le Premier ministre suédois, Göran Persson, avait reconnu que le PSB fait partie de la " grande famille socialiste ${ }^{25}$. Il a clôturé la campagne socialiste pour les législatives par un voyage en Allemagne.

Le PSB multiplie les contacts et la recherche de brevets extérieurs de légitimité afin de se construire une image de partenaire crédible sur le plan de l'intégration européenne. Les contacts avec les fédérations européennes des partis de gauche permettent au PSB de se présenter à la société comme un artisan possible de l'intégration européenne et " d'apporter un argument considérable pour contrer un des éléments essentiels de la propagande de l'UFD : "le PSB n'est pas un parti européen et en cours de réforme" $"{ }^{26}$. Părvanov a, quant à lui, défendu l'idée qu'aucun acteur politique ne doit monopoliser l'intégration européenne pour son usage propre ${ }^{27}$. De fait, la pluralité de l'Europe permet à chaque tendance politique bulgare d'y trouver des partenaires et des références. D'ailleurs, il est intéressant de noter que la priorité affectée aux relations internationales se traduit par la désignation du successeur de Părvanov à la tête du parti. Sergei Stanišev était précédemment en charge des relations extérieures du PSB et a, de ce fait, tissé des liens au sein de l'IS et du PSE, ainsi qu'avec d'autres partis de gauche.

Dès le premier congrès de rénovation du parti $\left(14^{\mathrm{e}}\right.$ Congrès extraordinaire du PCB), la nécessité de repenser la place du parti au sein du mouvement socialiste sur la base de nouveaux critères est affirmée. La poursuite des liens avec les partis communistes existants est maintenue, mais le Rapport politique envisage également l'activation des liens et de la collaboration avec l'Internationale socialiste et d'autres unions et forums de la gauche démocratique ${ }^{28}$. Quelques mois plus tard, lors du $39^{\mathrm{e}}$ Congrès du parti désormais renommé socialiste, l'orientation est réaffirmée : «le Parti socialiste bulgare exprime son désir de se joindre dans le cadre de son processus de rénovation à l'Internationale socialiste, de devenir un membre du mouvement mondial du

\footnotetext{
25 Părvanov (Georgi), “ Interview à la Télévision nationale ", 24 Časa, 15/05/01.

26 Sur l'activation des relations du PSB avec l'IS et le PSE, cf. Bulletin d'information du PSB, (12), novembre 2000, p. 8.

${ }^{27}$ Părvanov (Georgi), " Interview " (art. cit.).

28 " La rénovation du parti pour la construction d'une société démocratique socialiste en Bulgarie ", Rapport politique présenté au $14^{e}$ Congrès extraordinaire du PCB, 30 janvier - 2 février 1990, pp. 30-32.
} 
socialisme démocratique ${ }^{29}$. On constate la mise en place d'un couplage entre rénovation et adhésion à l'IS. Les PSB a déposé sa demande d'adhésion à l'IS en 1992, mais sa candidature posait problème à plusieurs titres : la lenteur de la transformation du parti et la présence de courants marxistes au sein du PSB ont renforcé la réticence initiale de l'IS à nouer des contacts avec les anciens partis communistes ${ }^{3 \circ}$. De surcroît, la Bulgarie comptait déjà un représentant dans l'Internationale, le Parti social-démocrate "historique", membre de plein droit. La victoire électorale de 1994 et l'alliance du PSDB avec l'UFD avaient commencé à vaincre les réticences de l'IS, mais les relations ont été gelées suite à l'échec du gouvernement socialiste et le départ de plusieurs réformateurs pour l'Euro-gauche. En effet, la crise de 1997 a accru l'isolement du parti qui, en perdant le pouvoir, perdait aussi des opportunités d'entretenir des contacts internationaux. Ce d'autant plus que parmi les réformateurs qui ont quitté le PSB en 1997 pour créer l'Euro-gauche se trouvent ceux qui menaient les négociations avec l'Internationale socialiste. Leur départ semblait répondre par la négative à la question de la capacité de transformation du parti. Le bilan est peu encourageant pour le PSB à l'issue de cette crise : le parti a subi une défaite sans précédent aux élections, ne peut plus accéder seul au pouvoir, il a perdu le monopole de la gauche et doit faire face à deux nouveaux concurrents - l'Eurogauche et le Bloc uni de travail - qui se réclament aussi de la social-démocratie européenne. Après les élections, le leader du PSB prend acte du " retrait visible d'une série de partenaires internationaux surtout parmi les partis sociaux-démocrates d'Europe de l'Ouest " ainsi que du fait " qu'une partie des contacts du PSB étaient menés par des hommes politiques qui ont rejoint l'Euro-gauche ${ }^{31}$. La nouvelle équipe à la tête du parti se pose comme objectif d'intensifier les relations avec l'IS et le PSE et, de fait, elle fait preuve de plus d'ouverture et de réceptivité à l'influence extérieure. Le dialogue avec l'IS reprend après la chute du gouvernement Videnov, mais c'est l'Euro-gauche qui a obtenu le statut d'observateur en 1999. Plus généralement, l'IS a refusé de désigner le parti social-démocrate authentique parmi tous ceux qui revendiquaient leur appartenance à cette famille et qui courtisaient l'organisation (l'Euro-gauche, le PSB et

\footnotetext{
29 " Plate-forme pour la rénovation ultérieure et la transformation du PSB en parti de gauche moderne du socialisme démocratique ", adoptée au cours du $39^{e}$ Congrès du PSB, septembre 1990
}

30 Pridham (Geoffrey), "Patterns of Europeanisation and Transnational Party Cooperation : Party Developement in Central and Eastern Europe ", Paper for the Workshop on European Aspects of PostCommunist Party Development, ECPR Sessions, University of Mannheim, 26-31 March 1999, p. 12 et Queval (Alex), " La coopération entre partis politiques. Le cas de l'Internationale socialiste en Europe centrale et orientale ", in Delwit (Pascal), Waele (Jean-Michel de), éds., La coopération paneuropéenne des partis politiques, Paris / Montréal : L'Harmattan, 1998 font état de cette réticence. Toutefois, elle a été vaincue pour certains anciens partis communistes admis au sein de l'IS au Congrès du 11 septembre 1997.

${ }^{31}$ Sur les résultats des élections anticipées et les objectifs à venir du PSB et de la Gauche démocrate, cf. " Rapport du Président du PSB lors du plénum du 4 mai 1997 n, Bulletin d'information du PSB, (7), mai 1997. 
le Bloc uni du travail, le Mouvement sociaux-démocrates) elle a encouragé, comme le PSE, la synergie entre ces partis. Toutefois, Părvanov, qui a assisté avec Stanišev au dernier congrès de l'IS qui s'est tenu en novembre 2001 à Saint-Domingue, se dit convaincu de l'adhésion du PSB à l'IS lors du prochain congrès de l'Internationale ${ }^{32}$.

Le PSE entretient des liens plus étroits avec le PSB. Il a été un des artisans du rapprochement de la gauche bulgare avec la rencontre de Thessalonique en août 199833. C'est sous son auspice que les leaders du PSB, du PSDB, du Bloc Uni du Travail (BUT) et de l'Eurogauche ont examiné le "modèle" tchèque d'arrivée au pouvoir et l'éventuelle formation d'une alliance sociale-démocrate. Krăstjo Petkov, leader du BUT, définit le PSE à la fois comme un "pont" et une "locomotive" pour la gauche bulgare 34 .

Les fondations - le Forum européen pour la démocratie et la solidarité, Friedrich Ebert, Jean Jaurès etc. - jouent également un rôle actif dans l'établissement de contacts entre le PSB, l'IS et le PSE. Ce sont des canaux de diffusion de modèles, de pratiques et de représentations. La fondation Friedrich Ebert joue un rôle important dans la diffusion d'idées, d'expertise et de savoir-faire, elle finance des projets de recherche et la publication et la traduction d'ouvrages. D'ailleurs, la réunion de Lesidren (14 novembre 1997) qui a réuni les différents leaders de la gauche bulgare et a constitué une première opération de comptage et d'identification de la gauche, et donc de l'opposition au gouvernement de l'UFD, a été organisée par la fondation F. Ebert. Les socialistes bulgares ont également tissé des liens avec certains partis "amis" comme le SPD ou le PASOK, et d'autres partis successeurs d'Europe centrale et orientale.

L'intensification de la coopération transnationale des partis et l'intérêt croissant pour le PSB semble attester de l'évolution du parti, elle renforce également l'idée que l'alternance ne peut se réaliser sans lui. L'élection du leader socialiste à la présidence de la République bulgare en novembre 2001 a consolidé la position du parti au sein de la gauche bulgare et sa crédibilité - renforçant l'espoir au sein du PSB que l'IS et le PSE opteront pour un parti "fort" susceptible de conquérir le pouvoir qui se social-démocratise, plutôt que pour un parti "authentiquement" social-democrate qui a peu de chances d'exercer le pouvoir 35 . En fait, la réactivation des relations avec l'IS et le PSE correspond non seulement à la relance du processus de rénovation au sein du PSB, mais également au renforcement de la contrainte externe en Bulgarie : un directoire fi-

${ }^{2}$ Sega, 15/12/o1 et " Déclaration lors de la session du 15/12/o1 " du $44^{e}$ Congrès du PSB.

33 Depuis cette réunion la presse désigne ces partis comme le " quatuor de Thessalonique ".

34 Sega, (35), 03-09/09/98.

35 Todorov (Antony), art. cit., p. 20. 
nancier (caisse d'émission) est mis en place en juillet 1997, le processus d'intégration de la Bulgarie dans l'UE se concrétise et se développe avec le début des négociations d'adhésion ce qui souligne la nécessité d'avoir la confiance des partenaires européens. Ce processus renforce l'intérêt des fédérations européennes de partis pour la Bulgarie. Elles cherchent à recruter de futurs alliés, procèdent à l'identification de leurs analogues et participent à leur transformation.

L'IS et le PSE stimulent les processus de coopération et d'unification des différents partis de gauche bulgares. Il est vrai que ce processus est également lié au constat consécutif aux législatives de 1997 qu'aucun parti de gauche ne peut emporter seul la majorité, mais le plaidoyer des euro-socialistes en faveur du rapprochement, voire de l'unification, joue un rôle fortement incitatif. Sous leur impulsion on passe d'une logique d'alliance à une logique d'unification. Les leaders des différents partis de gauche prennent acte, aux lendemains de la réunion de Thessalonique, que " le PSE n'a aucune intention d'entretenir des contacts avec quatre partis différents ${ }^{36}$. Ce processus de rapprochement ne met pas fin aux concurrences entre ces partis pour le rôle du leader au sein même de la gauche, ni même aux résistances face au processus d'unification promu par le PSE. Toutefois, cette diplomatie d'influence a favorisé la création en janvier 2001 de l'Union politique Nouvelle gauche. Elle regroupe quatre partis : le PSB, une branche du Parti social-démocrate 37 , le Bloc uni du travail et le Mouvement des sociaux-démocrates formé par des transfuges de l'Eurogauche. La création de la Nouvelle gauche constitue une avancée importante, ne serait-ce que du point de vue symbolique : les anciens concurrents ont accepté de se parler et envisagent de gouverner ensemble. La formule réunit en effet le PSB, parti successeur, avec des représentants de la social-démocratie "historique" et des transfuges de 1997, ce qui attesterait de la transformation du parti, le PSB devient un partenaire souhaitable. Elle ne constitue toutefois pas un acteur politique identifiable sur la scène politique bulgare. Pour les deux scrutins de 2001, les différentes composantes de la gauche bulgare ont formé une coalition électorale plus large : la Coalition pour la Bulgarie ${ }^{38}$ dans laquelle le PSB a joué un rôle central.

Le PSE souhaite la transformation rapide de la Nouvelle gauche en acteur politique - lors de leur mission d'étude effectuée en mars 2002, les leaders du

\footnotetext{
${ }^{36}$ Petkov (Krăstjo), " Interview n, Sega, (35), 01-08/og/98.

37 Le PSDB s'est scindé à la suite de la décision de son leader, Petar Dertliev (décédé depuis), de cesser le partenariat avec l'UFD. Il s'agit d'une fraction du parti favorable à cette autonomisation dirigée par Petar Agov.
}

$3^{8}$ Elle est composée de 16 formations, dont les 4 organisations membres de la Nouvelle gauche, des partenaires traditionnels du PSB (notamment des agrariens), ainsi que des organisations de citoyens, des mouvements des Roms bulgares. 
PSE ont clairement exprimé cet impératif - et commence à la considérer comme un interlocuteur. En septembre 2002, la Nouvelle gauche a obtenu une invitation à participer aux réunions de la Présidence du PSE ce qui s'apparente au statut de membre associé. Cette décision sera en vigueur jusqu'au prochain congrès du PSE en 2004 qui statuera définitivement. L'union sera représentée par le leader du PSB, Stanišev - ce qui semble confirmer le leadership du PSB au sein de la formation. Enfin, la direction du PSB espère obtenir une invitation à rejoindre l'IS lors de son prochain congrès. Părvanov a d'ailleurs déclaré en décembre 2001 que " le PSB et la gauche unie auront le soutien de l'Europe de gauche. Nous sommes à présent une partie organique de l'espace social-démocrate européen et mondial - et c'est un fait confirmé par les leaders de l'IS et du PSE "39. La relance du processus de rénovation et de social-démocratisation du PSB et l'activation des liens avec l'IS et le PSE tendent à attester de la normalisation du parti successeur - de sa sortie du ghetto post-communiste. Ce processus montre le rôle joué par les incitations et les contraintes européennes dans la transformation d'un parti. La mutation du PSB se traduit également par une réorientation de ses positions en matière de politique étrangère et principalement par son ralliement à l'adhésion à l'OTAN.

\section{LE PSB - UN PARTI DÉSIREUX / CAPABLE DE MENER LA BULGARIE SUR LE CHEMIN DE L'EUROPE ?}

Comme les autres pays du continent auparavant intégrés dans le camp socialiste, la Bulgarie a fait le choix du rapprochement avec l'Europe démocratique ${ }^{4}$. Il s'agissait de normaliser les relations diplomatiques avec les pays de l'ancien camp adverse puis, après la disparition des structures comme le CAEM et le Pacte de Varsovie, de trouver de nouveaux partenaires et alliés ce qui supposait également de redéfinir les rapports avec l'ancien tuteur, l'URSS, puis son successeur, la Russie. Les conflits dans les Balkans ont de surcroît souligné la nécessité d'assurer la sécurité de la Bulgarie dans un contexte régional instable. Dans ce contexte complexe, le retour vers l'Europe s'est imposé dès le début des changements comme une voie consensuelle, bien que non exclusive du maintien ou du développement de liens spéciaux avec la Russie et/ou les ÉtatsUnis. Le retour à l'Europe passe également pas une volonté d'intensification

39 Părvanov (Georgi), " Discours politique ", $44^{e}$ Congrès du PSB, session du 15/12/01.

$4^{\circ} \mathrm{Cf}$. notamment Dimitrov (Vesselin), Bulgaria. The uneven transition. London / New York : Routledge, 2001 ; Stoilov (Georgi), " Bulgaria and the West n, Peace and Security, 32 (1), janvier 2001.

${ }^{41}$ Jelev (Jeliou), op. cit, p. 46. 
des relations bilatérales. Ce que le Président Jelev a nommé la "stratégie des deux lignes parallèles " de l'adhésion bulgare à l'Europe : d'une part des actions en vue de son adhésion directe aux structures européennes et euro-atlantiques et d'autre part, la signature de traités bilatéraux avec tous les États européens ${ }^{41}$. Toutefois, la Bulgarie n'a pas réussi à nouer des liens privilégiés avec un pays particulier et n'a pas bénéficié d'un parrainage efficace.

La réorientation de la politique étrangère et de sécurité et principalement l'adhésion à l'OTAN a un statut de test pour la nouvelle démocratie bulgare et ses partis politiques - il s'agit encore une fois de prouver la rupture avec le passé, le changement de camp. L'enjeu est de taille pour la légitimation des acteurs politiques ce qui explique la force de la confrontation qui a pu prendre des formes assez violentes, les uns et les autres s'accusant mutuellement de vouloir sacrifier la Bulgarie aux intérêts étrangers ${ }^{42}$. Pour le PSB et l'UFD il s'agissait de s'imposer comme le principal - voire l'unique - promoteur de l'intégration euro-atlantique de la Bulgarie. Dans cette optique, le PSB en tant que parti successeur rattaché au maintien de relations fortes avec la Russie et hostile à l'OTAN avait le plus de difficultés et de réticences à s'imposer comme un artisan possible de cette intégration. Ce d'autant plus que son électorat était plutôt hostile à l'adhésion à l'OTAN. Ses adversaires politiques ont essayé de nier sa capacité à faire de la Bulgarie un pays européen en expliquant le rejet de l'OTAN par le fait que le PSB est le promoteur des intérêts russes en Bulgarie.

Au cours des premières années de changement, les quotidiens des partis Demokracia pour l'UFD et Duma pour le PSB - ont entretenu le débat sur l'allié stratégique en proposant des lectures du passé bulgare. L'étude des journaux menée par une équipe bulgare montre comment chaque journal propose son portrait de l'alliée bienveillant, qui oppose l'Europe et la Russie. " Duma construit un souvenir positif de la Russie (double libérateur), tentant de transformer ce souvenir en élément structurant de la mémoire collective et de légitimer ainsi ses positions actuelles dans le débat qui l'oppose à son concurrent politique sur la définition de la politique étrangère. Positions qui penchent en direction de l'orientation russophile qu'ils étayent toujours par un argument historique. " ${ }^{43}$ Ainsi, le passé était convoqué pour expliquer et orienter le présent et pour réactualiser les querelles entre russophiles et russophobes. En fait, comme le souligne Jeliou Jelev dans une interview en 1997, " le PSB comme l'UFD ont continué, les uns par pro-communisme et les autres par anti-communisme, à voir la Russie comme l'Union soviétique. (...) Le PSB n'est pas sorti

$4^{2}$ Cf. Monova-Galtier (Miladina), "Bulgarie : l'avenir par l'Europe et par l'OTAN ", Nouveaux mondes, (9), automne 1999, p. 268.

43 Deyanova (Liliana), et al., Nacionalnata identičnost v situacia na prehod : istoričeski resursi (L'identité nationale dans contexte de transition), Sofia : Minerva, 1997, p. 197. 
de l'ancien schéma communiste de l'éternelle amitié bulgaro-russe » 44 . L'instabilité politique du début de la période, l'extrême politisation des enjeux de politique étrangère, instrumentalisée dans les luttes politiques entre les deux grands partis politiques bulgares, et surtout la grave crise économique laissée par le gouvernement socialiste vont finalement repousser la Bulgarie dans une seconde vague de négociations, accroître son impréparation et son retard, lui imposant un rattrapage rapide et coûteux.

\section{Du consensus européen au consensus euro-atlantique}

Le couplage de l'adhésion à l'UE et à l'OTAN intervient en Bulgarie sous l'impulsion de l'UFD qui voit ainsi une façon de promouvoir son atlantisme et de souligner son rôle moteur dans ce double processus. Jeliou Jelev exprime bien cette idée : " il serait naif de croire qu'un pays postcommuniste pourrait être admis comme membre à part entière de l'Union européenne en refusant d'être candidat à l'OTAN $n 45$.

\section{Chronologie des relations de la Bulgarie avec l'UE et l'OTAN}

Les relations diplomatiques entre la Bulgarie et la CEE sont établies en août 1988. Le changement politique va permettre de renforcer les rapports entre la Bulgarie et les structures européennes : un accord commercial avec la CEE est conclu par le gouvernement socialiste de Lukanov en mai 1990, en septembre la Bulgarie est intégrée au programme PHARE et en décembre la Grande Assemblé nationale vote une résolution qui exprime le souhait de devenir membre de la CEE. L'intensification des liens se poursuit avec son adhésion au Conseil de l'Europe (mai 1992) et la conclusion d'un Accord d'association avec la CEE (mars 1993). En décembre 1995, la Bulgarie pose sa candidature à l'adhésion à l'Union européenne. La lenteur des réformes structurelles et l'instabilité politique bulgare suscitent des réserves de l'Union vis-à-vis de la candidature bulgare. Son examen à peine engagé, le pays fait face en 1997 à une nouvelle crise économique de grande ampleur, due au gouvernement socialiste, qui aboutira à une crise généralisée et à des élections anticipées. Il faut attendre les débuts de la stabilisation macro-économique et politique du pays, au deuxième semestre de l'année 1997, pour que les discussions soient relancées. Toutefois, dans son avis sur la candidature de la Bulgarie formulé en juillet 1997, la Commission a recommandé, sur la base des critères de Copenhague, que la Bulgarie ne fasse pas partie du premier groupe de pays avec lesquels des négociations devraient être ouvertes. Bien que l'avis ait reconnu que le pays se conformait aux critères politiques, il conclut que la

44 Jelev (Jeliou), entretien, 25/10/97 (menée par Magdalena Hadjiiski).

45 Jelev (Jeliou), op. cit, p. 69. 
Bulgarie n'avait réalisé que peu de progrès sur le plan économique. En mars 1998, se conformant à la demande des Quinze, la Bulgarie conclut avec l'UE un Partenariat pour l'adhésion. Sur la base du second rapport périodique de la Commission, la Bulgarie, ainsi que cinq autres pays, a été invitée au sommet d'Helsinki en décembre 1999 à entamer des négociations d'adhésion. Les négociations proprement dites ont commencé en mars 2000. En décembre 2001, le Conseil européen de Laeken a nommément cité les dix PECO jugés en mesure d'adhérer à l'Union en 2004, avant les prochaines élections au Parlement européen, ni la Bulgarie, ni la Roumanie ne figurent sur cette liste. En février 2002, le gouvernement bulgare - qui s'est fixé pour objectif d'adhérer à l'Union en 2007 - a adopté une stratégie visant à accélérer les négociations, craignant qu'après le "big bang" causé par l'entrée de dix nouveaux pays dans l'Union, celle-ci ne se prononce sur l'adhésion de la Bulgarie et de la Roumanie qu'après l'avancement de la candidature d'autres pays.

La question de l'adhésion éventuelle de la Bulgarie à l'OTAN est loin de faire l'unanimité. Certes, le gouvernement socialiste de Lukanov accueille favorablement l'invitation lancée aux PECO en juillet 1990 à établir des relations diplomatiques avec le pacte et des députés de la GNA déposent, en novembre 1990, une résolution en faveur de l'adhésion de la Bulgarie à l'OTAN. L'arrivée de l'UFD au pouvoir (1991) et les efforts du Président Jelev semblent favorables à l'intensification des relations entre la Bulgarie et l'OTAN. La Bulgarie fait partie du Conseil de coopération nord-atlantique créé en décembre 1991 pour institutionnaliser les liens entre l'OTAN et les PECO et rejoint, en 1994, le Partenariat pour la paix créé en 1993. En décembre 1993, l'Assemblée nationale adopte une déclaration en faveur de l'adhésion de la Bulgarie à l'OTAN et à l'UEO (elle devient membre associé de l'UEO en 1995). Cependant le rapprochement avec l'OTAN est freiné par l'arrivée au pouvoir des socialistes (décembre 1994). Il faudra attendre une nouvelle alternance en février 1997 pour que le dépôt officiel de la candidature bulgare à l'OTAN intervienne. Le Parlement nouvellement élu confirme ce choix en mai 1997 par l'adoption d'une déclaration que les députés socialistes ne votent pas. L'appui du gouvernement bulgare aux frappes aériennes contre la Serbie en mai 1999 (le gouvernement autorise l'accès de l'espace aérien bulgare aux avions de l'OTAN) marque l'intégration symbolique de la Bulgarie dans le camp atlantique. Une réforme d'envergure de l'armée bulgare est lancée pour se conformer aux critères de l'OTAN. Un consensus relatif sur l'intégration est obtenu en l'an 2000 et la Bulgarie a obtenu au cours du sommet de l'OTAN de novembre 2002 une invitation officielle à rejoindre l'organisation en 2004.

$\mathrm{Au}$ lendemain des changements, les documents du PSB prennent acte de la nécessité de redéfinir la politique étrangère de la Bulgarie dans le nouveau contexte des relations internationales. Malgré un consensus relatif sur l'inté- 
gration aux structures européennes et la nécessité de développer des relations avec les États-Unis, le PSB se distingue par son orientation pro-russe et de fortes réticences vis-à-vis de l'OTAN (le terme euro-atlantique est intégré dans les documents du PSB en 1994 sans renvoyer à un contenu spécifique) qui apparaissait en décalage avec la marche vers l'Europe entamée après 1989. Si la nécessité d'approfondir les relations avec la Russie a été aussi reconnue par l'UFD (1997), notamment parce que la Bulgarie dépendait des livraisons de gaz russe, le PSB était soupçonné de vouloir rétablir les relations paternalistes et déséquilibrées de l'époque de la grande amitié bulgaro-soviétique. L'étude des documents officiels du parti (programmes électoraux, rapports politiques, etc.) permet de dresser les caractéristiques principales de l'offre socialiste en matière de politique étrangère :

- tout d'abord l'accent est mis sur la nécessité du maintien de relations privilégiées avec l'URSS, puis la Russie. Les « liens traditionnels avec la Russie sont un énorme capital national, culturel et économique, garantie de notre survie nationale. Le PSB est pour le maintien et le développement des relations politiques, économiques et spirituelles avec l'Union soviétique ". La coopération avec l'URSS et les autres alliés est définie comme une priorité de la politique étrangère de la Bulgarie (1990). Cet accent sera progressivement atténué, mais le PSB veille toujours à se prononcer pour l'équilibre des relations avec les États-Unis et la Russie, alors que l'UFD est plus atlantiste. En fait, le gouvernement socialiste a été soupçonné de vouloir maintenir la Bulgarie dans la zone d'influence russe, notamment en s'alignant sur les positions russes vis-à-vis de l'OTAN. La perspective de la conclusion d'un accord de rapprochement entre la Russie et la Bulgarie en 1996 a provoqué de nombreuses craintes et la réaction du Président Jelev;

- on note également des nuances au sujet de la politique balkanique - le PSB est plutôt favorable à une neutralité, alors que l'UFD a soutenu les actions de l'ONU en ex-Yougoslavie.

Ces caractéristiques donnent l'impression que le PSB est tourné vers l'Est et le Sud, c'est-à-dire vers le passé. Impression corroborée par le gouvernement de Videnov, dont l'action a contribué à l'isolement de la Bulgarie. Le Premier ministre a entretenu des rapports tendus avec les bailleurs de fonds et particulièrement avec le FMI. Il s'est aussi confronté à plusieurs reprises avec le Président Jelev au sujet de la politique étrangère ${ }^{46}$. 
La principale différence entre les deux grands partis bulgares concerne l'adhésion à l'OTAN. En matière de sécurité, le PSB d'abord partisan du maintien de la participation de la Bulgarie au Pacte de Varsovie (programme électoral de 1990), se déclare en faveur de la création d'un " système effectif de sécurité et de coopération d'un nouveau type en Europe ". Certes, suite à la disparition du Pacte de Varsovie, le PSB réoriente son discours en matière de sécurité mais, l'inflexion inscrite dans le programme d'action du parti et la plateforme électorale de 1994, consiste en fait à envisager une éventuelle coopération de la Bulgarie avec l'OTAN, mais pas une adhésion. Le refus de l'adhésion à l'OTAN est exprimé à travers la promotion d'un nouveau système de sécurité et de coopération en Europe, le refus d'adhérer à des blocs militaires et la promotion de la neutralité de la Bulgarie.

Jusqu'en 1994, les réticences de l'OTAN à lancer un processus d'élargissement permettent au débat de rester ouvert, mais quand ce processus est lancé, la Bulgarie doit prendre position. Or, le PSB qui a remporté une majorité absolue aux élections législatives de décembre 1994 n'est pas prêt à franchir ce cap et élude la question. La proposition de déclaration parlementaire en faveur d'une adhésion de la Bulgarie à l'OTAN proposée par l'opposition n'a jamais pu être discutée 47 . Le gouvernement Videnov, sollicité par l'OTAN au cours de l'été 1996, ne dépose pas une demande d'adhésion.

La première tentative d'élaborer une position officielle du parti vis-à-vis de l'OTAN n'intervient qu'en 1998, lors d'un plénum (24 mars), le Conseil supérieur du PSB se prononce en faveur de la neutralité de la Bulgarie et propose que la question de l'adhésion éventuelle de la Bulgarie à l'OTAN soit tranchée par voie de référendum. La décision réaffirme la nécessité de trouver de nouvelles formes de sécurité dans le cadre de l'ONU pour assurer la sécurité de la région, elle souligne les risques inhérents à l'installation de bases militaires dans le pays et l'impossibilité de prendre en charge les efforts financiers nécessaires à l'adhésion. Cette position est maintenue lors $d u 43^{\mathrm{e}}$ Congrès du parti (mai 1998) qui réaffirme le refus de participer à des unions militaires (l'OTAN est définie comme telle) et à des opérations militaires. En fait, les socialistes craignent de mettre un terme aux liens traditionnels avec la Russie dont le PSB se veut le garant - tout en ayant conscience de la nécessité d'assurer la sécurité du pays. Ils pointent les dangers inhérents aux choix entre deux grandes puissances en rappelant les deux grandes " catastrophes nationales " qui ont succédé à la rupture de la neutralité bulgare durant les deux Guerres mondiales - insistant sur la nécessité de garder une neutralité, alors même que la Bulgarie n'a pas les moyens d'entretenir une armée suffisamment puissante pour assurer seule sa sécurité. D'ailleurs, malgré sa longue opposition à 
l'élargissement de l'OTAN, la Russie ne semblait ni apte ni désireuse d'assurer la sécurité de la Bulgarie. Le refus de prendre position en faveur de l'OTAN a également été motivé par la crainte de raviver des conflits au sein même du PSB où les courants sont majoritairement opposés à l'adhésion (le seul courant favorable à l'adhésion est le Mouvement pour l'unité et le développement du PSB plus connu comme " mouvement des généraux ", cf. documents proposés au $43^{\mathrm{e}}$ Congrès). Le PSB s'est violemment opposé à l'intervention aérienne menée par l'OTAN contre la Yougoslavie (mars - juin 1999) et à la décision de la majorité d'ouvrir l'espace aérien bulgare aux avions de l'alliance (vote contre). "L'agression militaire de l'OTAN contre un pays européen souverain fut une sérieuse erreur politique. (...) Le PSB s'est déclaré contre cette guerre. „48 Toutefois, au cours du même congrès et sans qu'il y ait eu pour autant un consensus au sein du parti, le principe de l'adhésion à l'OTAN est voté : " l'intégration européenne et euro-atlantique dans toutes les structures politiques, économiques et de défense militaire sera un objectif prioritaire pour le PSB aussi dans l'avenir. La question de l'adhésion à l'OTAN doit être tranchée par voie de référendum "49. L'exigence de référendum préalable à l'adhésion a été maintenue, mais en cas de consultation de ce type, la direction du parti semble prête à favoriser le vote positif plutôt que la neutralité. Evgueni Daïnov écrit dans Demokracia qu'en rejoignant les partisans de l'OTAN, le PSB perd l'unique spécificité qui le classait parmi les partis russophiles.

L'UFD a mis en doute la sincérité de ce ralliement après l'opposition du PSB aux frappes de l'OTAN en Yougoslavie. Cependant, le PSB n'est pas revenu sur cet engagement. En mai 2000, la majorité (trois députés votent contre) du groupe parlementaire socialiste vote en faveur de la position du Gouvernement sur les négociations avec l'Union européenne sur les thèmes de la politique étrangère et de sécurité : " parallèlement à son intégration à l'UE, la Bulgarie aspire à devenir membre de l'OTAN ". L'engagement est contenu dans les plates-formes pour les élections législatives et présidentielles de 2001 et depuis son élection à la Présidence de la République, Părvanov réaffirme sans cesse qu'il s'agit d'une priorité pour le pays. Les programmes électoraux pour les élections législatives et présidentielles de 2001 insistent également sur la continuité de la politique étrangère. La plate-forme politique adoptée au cours $d u 45^{\mathrm{e}}$ Congrès du PSB (juin 2002) se déclare en faveur de l'adhésion à l'OTAN qui est définie comme une priorité et s'engage à œuvrer en ce sens - elle note également la continuité des priorités internationales et stratégiques (adhésion à l'UE et à l'OTAN). Le nouveau Président a effectué son premier voyage officiel à

$4^{8}$ " Bilan de l'action du Conseil supérieur du PSB dans la période entre le $43^{\mathrm{e}}$ et le $44^{\mathrm{e}}$ Congrès ", document présenté lors du $44^{e}$ Congrès (mai 2000), p. 15.

49 " Stratégie nationale pour le développement. Travail, croissance, développement ", adoptée au cours du $44^{e}$ Congrès, mai 200o, p. 35. 
Bruxelles, Strasbourg et a visité le commandement de l'OTAN (février 2002). Il a créé un Conseil de l'intégration euro-atlantique et s'est entouré dans le domaine de conseillers réputés pour leur ouverture internationale. D'ailleurs, le PSB développe une stratégie plus active en faveur de l'intégration européenne à partir des années 1999-2000, plusieurs réunions sont consacrées à la question. En mars 2000, un Conseil de l'intégration européenne est créé auprès du Conseil supérieur du PSB. Il s'agit de prendre ainsi une part plus active à ce processus de modernisation et de changement social sans précédent. Il s'agit également de former les cadres aux questions européennes (notamment les élus locaux) et de sensibiliser les militants à ces questions ${ }^{50}$. Par le biais du processus d'intégration, le PSB entend promouvoir avec l'appui de ses " amis " européens une vision plus sociale de l'Europe, ce qui permet de réhabiliter le discours social en le déconnectant du passé communiste.

Le ralliement du PSB à la candidature bulgare à l'OTAN a été imposée par la direction qui semble avoir pris acte de l'absence d'alternative (ce d'autant plus que la candidature du pays avait été déposée) et de l'impossibilité de prétendre au pouvoir sans prendre favorablement position sur ce sujet. Le ralliement est justifié de façon pragmatique : l'OTAN est le seul système collectif de sécurité en Europe et la " Bulgarie pourra mieux défendre ses intérêts nationaux en étant dans le traité de l'Atlantique nord qu'en dehors " et présenté comme une " étape naturelle dans le développement et l'évolution du PSB sur cette question ${ }^{51}$. Dans ce cas la contrainte externe - l'Europe le veut et la Bulgarie n'a pas les moyens de s'y opposer - a joué un rôle important pour imposer la décision au sein du parti. Le cap euro-atlantique résulte d'un choix pragmatique et les socialistes promeuvent une vision " euro-réaliste " du processus d'élargissement qui contraste avec la vision émotionnelle véhiculée par l'UFD tout au long des années 1990. Ce qui correspond également à la normalisation de la vie politique bulgare et au progressif dépérissement du clivage ex-communiste / anticommuniste. Le ralliement euro-atlantique du PSB a contribué à banaliser ces priorités. Elles se prêtent moins aisément à une instrumentalisation qui vise à transformer l'Europe en ligne de démarcation susceptible de faire perdurer le clivage communiste / anticommuniste. Le PSB commence à obtenir le soutien de partenaires extérieurs et devient un allié crédible sur la scène politique bulgare - reste à confirmer cette transformation dans la pratique gouvernementale.

$5^{\circ}$ Dimitrov (Georgi), « Prisăedinjavaneto na Bălgaria kăm Evropejskija săjuz i pozicijata na Bălgarskata socialističeska partija " (L'intégration de la Bulgarie a l'UE et la position du PSB), Novo vreme, novembre 2000. L'auteur est coordonnateur du Conseil de l'intégration européenne du PSB.

51 Părvanov (Georgi), « Discours politique ", art. cit. 